\title{
The Need for Preclinical Biomarkers in Systemic Autoimmune Rheumatic Diseases
}

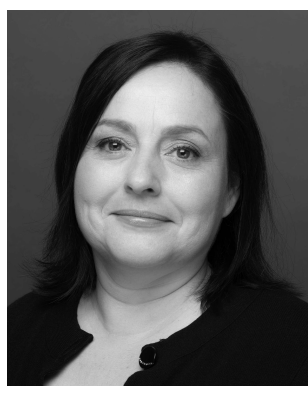

Systemic autoimmune rheumatic diseases (SARD) include systemic lupus erythematosus (SLE), Sjögren syndrome (SS), rheumatoid arthritis (RA), and systemic sclerosis (SSc). SARD are characterized by immune dysregulation, with varying commonalities in genetic susceptibility ${ }^{1,2}$, peripheral blood cytokine profiles (e.g., interferon- $\alpha)^{3}$ and the production of antinuclear antibodies (ANA). Each clinical syndrome is uniquely defined by the presence of specific autoantibodies, target organ predilection, response to therapy, and longterm prognosis.

Although the precise pathogenic mechanisms underlying SARD in general, and the development of specific clinical entities in particular are largely unknown, they likely follow a common pattern of progression from an early preclinical stage through benign to pathologic autoimmunity, culminating in overt clinical disease. Several lines of evidence support this paradigm, with immunological derangements including time-dependent expansion of pathogenic autoantibodies (e.g., $\mathrm{RA}^{4}, \mathrm{SLE}^{5}$ ), discrete cellular abnormalities (e.g., decreased regulatory $\mathrm{T}$ cells), and rising levels of circulating proinflammatory cytokines (e.g., $\mathrm{RA}^{6}$ ) noted in patients with SARD prior to diagnosis. This temporal framework is likely common to all SARD; however, variation in the progression of individual cases - for example with regard to the elaboration of specific autoantibodies and other cellular and biochemical derangements - likely influences the eventual manifestation of particular clinical syndromes. The timing and nature of disease-defining variables and branch points have yet to be elucidated.

Autoantibodies can be found in patients with SARD years before the emergence of clinical disease, but they are insufficient to identify individuals at risk of progression. Serological abnormalities can be found in individuals unlikely to develop SARD, such as first-degree relatives of patients with SARD, or individuals with organ-specific autoimmune disease. Further, given the extended time interval between the development of serological abnormalities and clinically apparent SARD, autoantibodies cannot predict the timing of disease onset. A recent study of preclinical RA defines a 2-year period of escalating immune dysregulation marked by autoantibody epitope spreading and rising inflammatory cytokines preceding the onset of clinical arthritis ${ }^{4}$. These observations are likely applicable to other SARD, with the immediate preclinical period characterized by the rapid accrual of multiple biochemical abnormalities that frame the transition from preclinical to overt disease. The identification of disease-specific biomarkers within this period of accelerated immune derangement offers the opportunity to identify "at-risk" patients, and potentially intervene prior to the development of full-blown SARD.

In this issue of The Journal, Steen and co-workers report on a novel circulating microRNA (miRNA) signature that identifies patients with SSc when compared to healthy controls (HC) and, more important, discriminates between patients with established SSc and $\mathrm{SLE}^{7}$. These miRNA are short ( 22 nucleotides), highly conserved, non-coding sequences that play a critical role in the posttranslational modulation of gene expression ${ }^{8}$. They regulate most protein-coding genes, exerting control through binding to target messenger RNA (a single miRNA can have multiple targets), resulting in repression of protein expression or direct degradation of the target message. MiRNA play a critical role in cellular homeostasis ${ }^{8}$, and their aberrant expression has been implicated in the pathogenesis of autoimmune conditions including $\mathrm{RA}^{9}, \mathrm{SLE}^{10}$, and multiple sclerosis ${ }^{11}$. Further, miRNA likely modulate disease severity because expression levels of specific miRNA correlate with measures of disease activity in $\mathrm{RA}^{12}$ and $\mathrm{SLE}^{13}$. Mature miRNA are expressed within tissues but can also be found in stable forms in the circulation, raising the possibility that they may serve as accessible biomarkers of disease ${ }^{14}$.

MiRNA are key regulators of tissue growth and repair, and profound alterations in miRNA expression are associated with pulmonary ${ }^{15}$, renal ${ }^{16}$, and postinfarct $\operatorname{cardiac}^{17}$ fibrosis. Because tissue fibrosis is the defining clinical feature of SSc, Steen, et al appropriately examined the expression profile of miRNA implicated in

See Micro-RNA in SSc, page 214

Personal non-commercial use only. The Journal of Rheumatology Copyright (c) 2015. All rights reserved. 
SSc-associated fibrosis, contrasting their expression to transcripts linked to SLE and inflammation. From an initial screen of 45 transcripts the authors identified 26 promising candidates, excluding miRNA with poor reproducibility, miRNA expressed in a limited number of patients, or miRNA compromised by sample age. This refined dataset was systematically tested to define the optimal combination of miRNA that yielded the best discriminatory accuracy between SSc and both HC and SLE, and further between SSc and HC, and the 2 disease states (SSc vs SLE). From this analysis the authors identified a 21-miRNA signature that discriminates between SSc and SLE and HC; they also defined miRNA profiles that distinguish between SSc and HC (22-miRNA panel), and SSc and SLE (14-miRNA panel) with diagnostic accuracies of 0.95 and 0.84 , respectively. Further refinement demonstrated that miR-17 92 miRNA cluster (decreased in SSc) was the main contributor to the miRNA profile discriminating between SSc and HC. It is of note that increased expression of the miR-17 92 cluster has been shown to reduce fibrosis ${ }^{18}$, suggesting that these findings reflect underlying disease pathology, and highlighting the suitability of this cluster as a disease-specific biomarker. A second signature comprising 4 transcripts, miR-142-3p and miR-223 (decreased in SSc) and mIR-150 and miR-638 (increased in SSc), effectively differentiated between SSc and SLE without a significant loss of diagnostic accuracy (0.81 vs 0.84$)$. The authors propose that the miR-142-3p is of particular relevance because it is directly implicated in the regulation of transforming growth factor- $\beta$, a primary driver of tissue fibrosis ${ }^{19}$, again anchoring their discriminatory signature in disease pathogenesis.

The results reported in this study add to a short but growing list of potential disease-defining biomarkers that discriminate between closely related SARD. In a ground-breaking study, circulating CXCL4 [chemokine (C-X-C motif) ligand 4 or platelet factor 4] was shown to be significantly elevated in patients with early diffuse SSc versus patients with established disease and SLE patients ${ }^{20}$. It remains to be determined whether this disease specificity also discriminates between SSc and other SARD. Differences in the peripheral blood expression of BAFF (B cell activating factor, B lymphocyte stimulator), a cytokine contributing to the rescue of autoreactive B cells, has been noted between distinct SARD, with only $\sim 20 \%$ of patients with $\mathrm{RA}^{21}$ showing elevated levels in contrast to the majority of both patients with SLE and $\mathrm{SS}^{22,23}$. Although the use of candidate SARD-specific biomarkers has not been well studied in patients in the preclinical stage, it is reasonable to expect that several of these discriminatory abnormalities will be present in the predisease phases of disease evolution.

The identification of predisease biomarkers and the ability to stratify patients by risk of progression and the potential development of a specific clinical syndrome presents a significant therapeutic opportunity. Introduction of therapy in the preclinical stage has the potential not only to limit organ involvement and dysfunction, but also to attenuate or inhibit disease progression. Support for early proactive intervention comes from studies of patients with SARD at the cusp of disease development. It has been noted that introduction of hydroxychloroquine is associated with later onset of SLE in individuals with stereotypical serology ${ }^{24}$, and treatment of RA within 12 weeks of diagnosis was significantly associated with disease remission ${ }^{25}$. Authors from this latter study concluded that this small window of time following symptom onset and diagnosis represented the most significant opportunity to establish RA quiescence.

Early intervention would be of particular relevance in SSc because, to date, no therapy has been shown to be effective in reversal or attenuation of fibrosis. The proposed use of a fibrotic-specific miRNA signature offers the promise of pre-SARD diagnosis prior to the development of clinically significant parenchymal involvement, and also presents a potential target for therapeutic intervention. This is not an unrealistic proposal, because anti-miRNA targeting strategies have already proven both safe and effective in the treatment of hepatitis C infection ${ }^{26}$.

CAROLINA LANDOLT-MARTICORENA, MD, PhD, FRCPC,

University Health Network,

Toronto Western Hospital Research Institute,

Department of Medicine and Division of Rheumatology,

University of Toronto,

Toronto, Ontario, Canada.

Address correspondence to Dr. C. Landolt-Marticorena, Toronto Western Hospital, 399 Bathurst St., Toronto, Ontario M5T 2S8, Canada.

E-mail: Dr.Carol.Landolt-Marticorena@uhn.on.ca

\section{REFERENCES}

1. Martin JE, Assassi S, Diaz-Gallo LM, Broen JC, Simeon CP, Castellvi I, et al. A systemic sclerosis and systemic lupus erythematosus pan-meta-GWAS reveals new shared susceptibility loci. Hum Mol Genet 2013;22:4021-9.

2. Kottyan LC, Zoller EE, Bene J, Lu X, Kelly JA, Rupert AM, et al. The IRF5-TNPO3 association with systemic lupus erythematosus has two components that other autoimmune disorders variably share. Hum Mol Genet 2014; Sept 8 (E-pub ahead of print).

3. Crow MK. Type I interferon in organ-targeted autoimmune and inflammatory diseases. Arthritis Res Ther 2010;12 Suppl 1:S5.

4. Sokolove J, Bromberg R, Deane KD, Lahey LJ, Derber LA, Chandra PE, et al. Autoantibody epitope spreading in the pre-clinical phase predicts progression to rheumatoid arthritis. PLoS One 2012;7:e35296.

5. Arbuckle MR, McClain MT, Rubertone MV, Scofield RH, Dennis GJ, et al. Development of autoantibodies before the clinical onset of systemic lupus erythematosus. N Engl J Med 2003;349:1526-33.

6. Kokkonen H, Soderstrom I, Rocklov J, Hallmans G, Lejon K, Rantapaa Dahlqvist S. Up-regulation of cytokines and chemokines predates the onset of rheumatoid arthritis. Arthritis Rheum 2010;62:383-91.

Personal non-commercial use only. The Journal of Rheumatology Copyright @ 2015 . All rights reserved. 
7. Steen SO, Iversen LV, Carlsen AL, Burton M, Nielsen CT, Jacobsen $\mathrm{S}$, et al. Circulating cell-free microRNA profile in systemic sclerosis is distinct from both healthy controls and systemic lupus erythematosus. J Rheumatol 2015;42:214-21.

8. Xiao C, Rajewsky K. MicroRNA control in the immune system: basic principles. Cell 2009;136:26-36.

9. Chatzikyriakidou A, Voulgari PV, Georgiou I, Drosos AA. miRNAs and related polymorphisms in rheumatoid arthritis susceptibility. Autoimmunity Rev 2012;11:636-41.

10. Stagakis E, Bertsias G, Verginis P, Nakou M, Hatziapostolou M, Kritikos $\mathrm{H}$, et al. Identification of novel microRNA signatures linked to human lupus disease activity and pathogenesis: miR-21 regulates aberrant $\mathrm{T}$ cell responses through regulation of PDCD4 expression. Ann Rheum Dis 2011;70:1496-506.

11. Du C, Liu C, Kang J, Zhao G, Ye Z, Huang S, et al. MicroRNA miR-326 regulates $\mathrm{TH}-17$ differentiation and is associated with the pathogenesis of multiple sclerosis. Nat Immunol 2009;10:1252-9.

12. Murata $\mathrm{K}$, Yoshitomi $\mathrm{H}$, Tanida $\mathrm{S}$, Ishikawa M, Nishitani $\mathrm{K}$, Ito $\mathrm{H}$, et al. Plasma and synovial fluid microRNAs as potential biomarkers of rheumatoid arthritis and osteoarthritis. Arthritis Res Ther 2010;12:R86.

13. Singh RP, Massachi I, Manickavel S, Singh S, Rao NP, Hasan S, et al. The role of miRNA in inflammation and autoimmunity. Autoimmunity Rev 2013;12:1160-5.

14. Weiland M, Gao XH, Zhou L, Mi QS. Small RNAs have a large impact: circulating microRNAs as biomarkers for human diseases. RNA Biol 2012;9:850-9.

15. Pandit KV, Corcoran D, Yousef H, Yarlagadda M, Tzouvelekis A, Gibson KF, et al. Inhibition and role of let-7d in idiopathic pulmonary fibrosis. Am Journal Respir Crit Care Med 2010;182:220-9.

16. Li R, Chung AC, Dong Y, Yang W, Zhong X, Lan HY. The microRNA miR-433 promotes renal fibrosis by amplifying the TGF-beta/Smad3-Azin1 pathway. Kidney Int 2013;84:1129-44.

17. Wang J, Huang W, Xu R, Nie Y, Cao X, Meng J, et al. MicroRNA-24 regulates cardiac fibrosis after myocardial infarction. J Cell Mol Med 2012;16:2150-60.
18. Dakhlallah D, Batte K, Wang Y, Cantemir-Stone CZ, Yan P, Nuovo $\mathrm{G}$, et al. Epigenetic regulation of miR-17 92 contributes to the pathogenesis of pulmonary fibrosis. Am J Respir Crit Care Med 2013; 187:397-405.

19. Lamouille S, Xu J, Derynck R. Molecular mechanisms of epithelial-mesenchymal transition. Nat Rev Mol Cell Biol 2014;15:178-96.

20. van Bon L, Affandi AJ, Broen J, Christmann RB, Marijnissen RJ, Stawski L, et al. Proteome-wide analysis and CXCL4 as a biomarker in systemic sclerosis. N Engl J Med 2014;370:433-43.

21. Vallerskog T, Heimbürger M, Gunnarsson I, Zhou W, Wahren-Herlenius M, Trollmo C, et al. Differential effects on BAFF and APRIL levels in rituximab-treated patients with systemic lupus erythematosus and rheumatoid arthritis. Arthritis Res Ther 2006;8:R167.

22. Landolt-Marticorena C, Wither R, Reich H, Herzenberg A, Scholey $\mathrm{J}$, Gladman DD, et al. Increased expression of B cell activation factor supports the abnormal expansion of transitional B cells in systemic lupus erythematosus. J Rheumatol 2011;38:642-51.

23. Vincent FB, Saulep-Easton D, Figgett WA, Fairfax KA, Mackay F. The BAFF/APRIL system: emerging functions beyond B cell biology and autoimmunity. Cytokine Growth Factor Rev 2013;24:203-15.

24. James JA, Kim-Howard XR, Bruner BF, Jonsson MK, McClain MT, Arbuckle MR, et al. Hydroxychloroquine sulfate treatment is associated with later onset of systemic lupus erythematosus. Lupus 2007;16:401-9.

25. Gremese E, Salaffi F, Bosello SL, Ciapetti A, Bobbio-Pallavicini F, Caporali R, et al. Very early rheumatoid arthritis as a predictor of remission: a multicentre real life prospective study. Ann Rheum Dis 2013;72:858-62.

26. Janssen HL, Reesink HW, Lawitz EJ, Zeuzem S, Rodriguez-Torres $\mathrm{M}$, Patel K, et al. Treatment of HCV infection by targeting microRNA. N Engl J Med 2013;368:1685-94.

J Rheumatol 2015;42:152-4; doi:10.3899/jrheum.141366 